
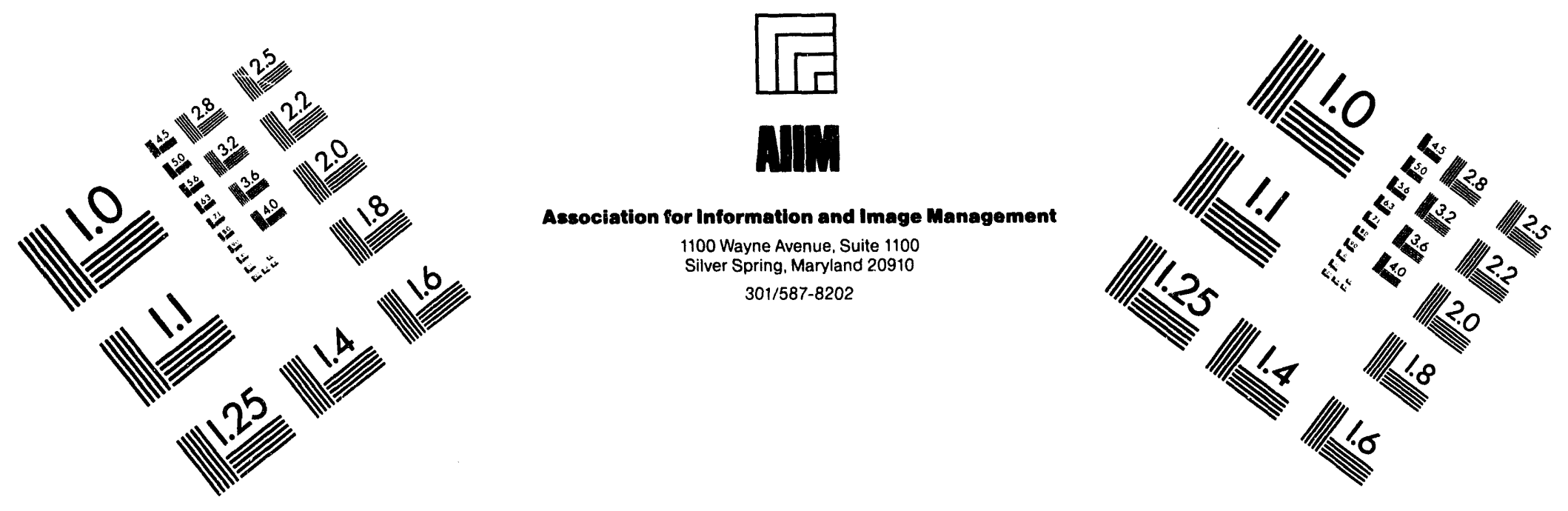

\title{
Centimeter
}

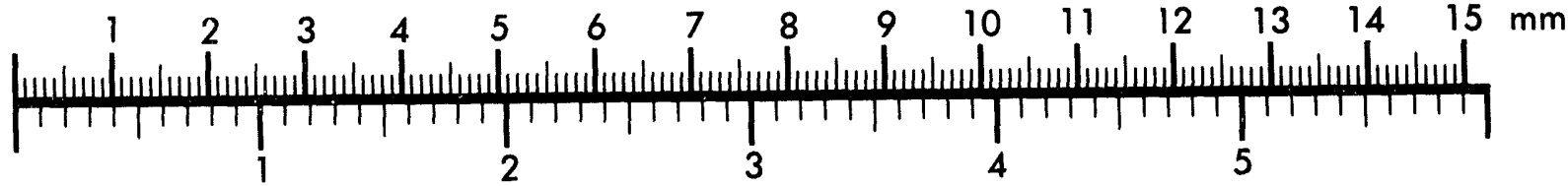

Inches
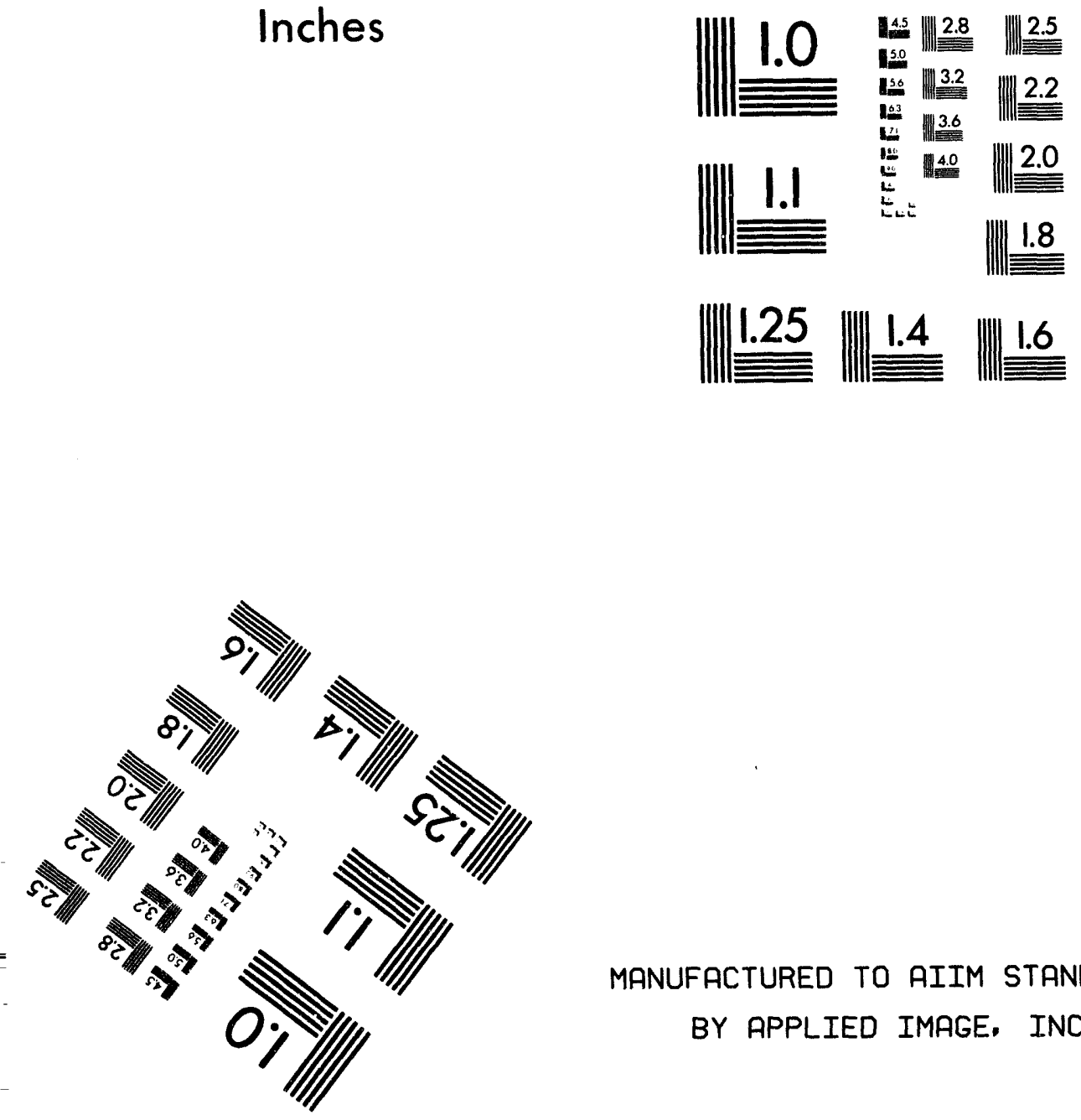

MANUFACTURED TO AIIM STANDARDS

BY APPLIED IMAGE. INC.

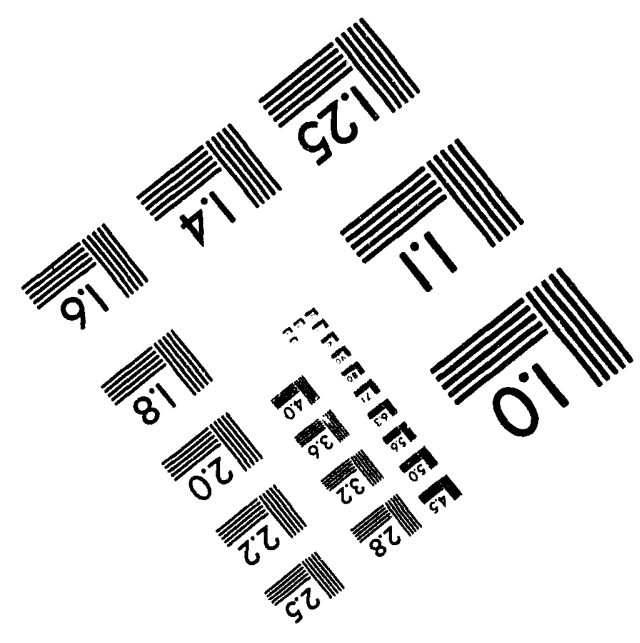



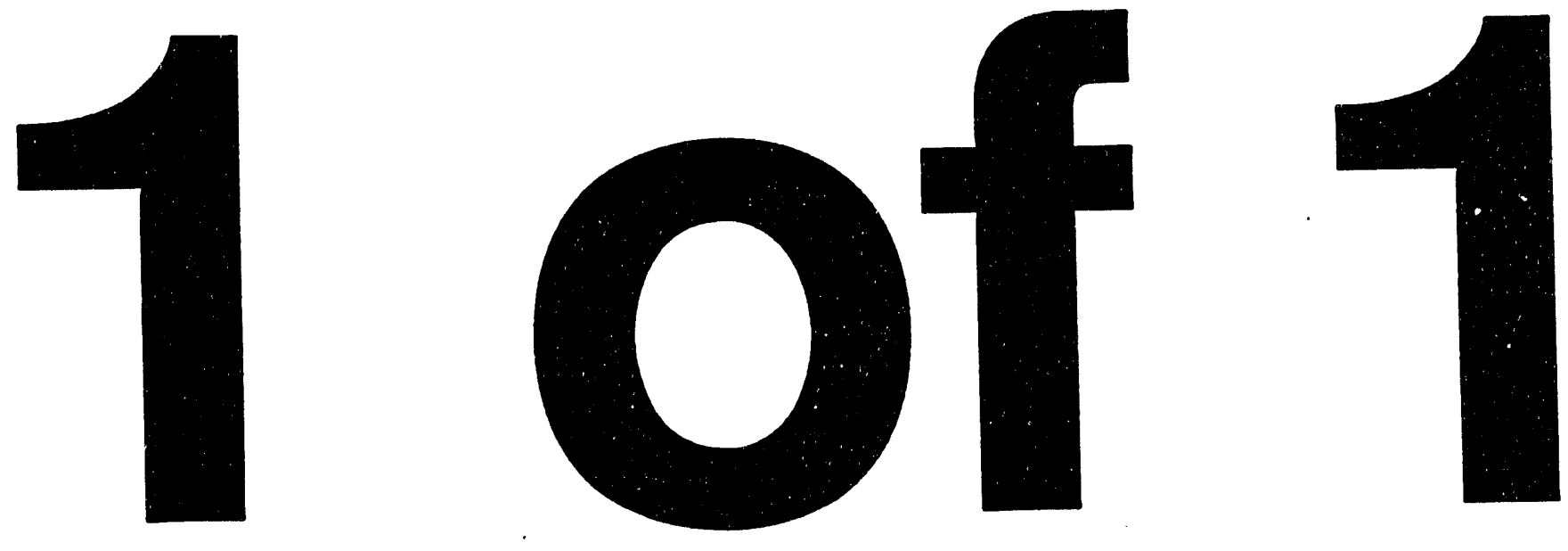


\section{PERFORMANCE OF PHOTON POSITION MONITORS AND STABILITY OF UNDULATOR BEAMS AT THE ADVANCED LIGHT SOURCE}

Tony Warwick, Nord Andresen, Greg Portmann and Alan Jackson

Advanced Light Source

Accelerator and Fusion Research Division

Lawrence Berkeley Laboratory

University of California

Berkeley,CA 94720

July 15, 1994

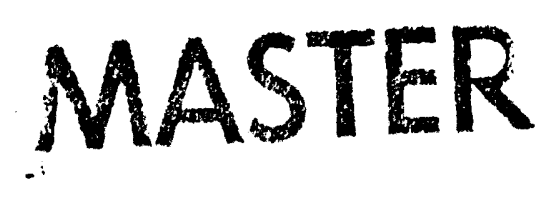

"This work was supported by the Director, Office of Energy Research, Office of Basic Energy Sciences, Materials Sciences Division of the U.S. Department of Energy, under Contract no. DE-AC03-76SF00098 
Performance of Photon Position Monitors and Stability of Undulator Beams at the Advanced Light Source

Tony Warwick, Nord Andresen, Greg Portmann and Alan Jackson. Lawrence Berkeley Laboratory, Berkeley, CA 94720, USA

Position monitors are implemented in three undulator beamlines at the ALS. Their performance has been studied carefully on one of these lines and is reviewed. The monitors work as expected and show the ALS to be an exceptionally stable source of synchrotron radiation.

\section{INTRODUCTION}

Synchrotron light sources in the past have had electron beams that move around and adversely affect experiments. Sensitive position monitors have been developed for undulator beams to anticipate this problem at the ALS. The design follows a conventional approach ${ }^{1}$ of sensing differences of currents from photo-emissive blades which protrude transversely into the undulator beam. Two monitors are in use on ALS undulator beamline 7.02 and their sensitivity to beam motion and to changes of the undulator gap will be discussed. They have shown that the ALS storage ring is exceptionally stable. Because of the intrinsic stability of the facility the systems planned for orbit position feedback are not urgently needed. The monitors will be incorporated into the storage ring control system gradually, for use in feedback as required. They presently act as precise diagnostics for beam position shifts and drift.

\section{MONITOR DESIGN}

These monitors work in pairs, one at $8.5 \mathrm{~m}$ distance from the center of the undulator straight inside the shield wall, the other at $12.5 \mathrm{~m}$ distance, outside the wall. The position of the photon beam is measured at each location and projected back to the center of the undulator. The first monitor measures only the vertical position of the beam with two blades protruding into the radiation from above and from below. The second has four blades at $45^{\circ}$, to stay out of the shadow of the first monitor and to measure the horizontal and the vertical position. The blades are separated transversely by $2.5 \mathrm{~mm}$. They protrude into the $1 / \gamma$ zone of high power radiation but not into the diffraction limited undulator central cone. This ensures large signals (hundreds of $\mu \mathrm{A}$ ) and relative insensitivity to the light from the bend magnet fringe fields.

The vertical stability is usually the critical parameter. As a pair, the monitors can detect vertical position and angle fluctuations of the electron orbit at the center of the undulator with a resolution of $2 \mu \mathrm{m}$ and $2 \mu \mathrm{rad}$ respectively. In the horizontal direction the second monitor provides angular information. 
The structure to cany the blades is carefully engineered. The cooled copper support is symmetric above and below the beam and is mounted on a water filled post with brackets made of 'super-invar'. This reduces the motion of the monitor with respect to the floor, due to environmental temperature changes, to a negligible level. Best measurements of the stability of this structure have shown motion less than $1 \mu \mathrm{m}$.

The blades themselves are tapered towards the source so that light falls at grazing incidence not only on the leading edge, but also down both sides. This reduces the sensitivity to angular alignment in the beam. Figure 1 . shows the monitor. The blades are mounted between clearing electrodes, normally biased to $+75 \mathrm{~V}$. The upper blades are upstream and the lower blades are downstream of the support so that there is no exchange of electrons vertically between the blades. This is a provision in case the detector is to be operated reverse-biased 3 to inhibit its response to low energy photons. So far this has not been necessary. The heating of the blades in the beam at maximum power, with an undulator deflection parameter of $K=4$ ( 20 Watts absorbed per blade) is computed to cause a temperature rise of no more than $50^{\circ} \mathrm{C}$ at the tip and a deformation of no more than $4 \mu \mathrm{m}$.

\section{ELECTRONICS}

Most of the tests reported in this paper were made using Keithley model 486 picoammeters. Amplifier stability was checked with a battery, which simulated the currents from the blades to measure the effective position error from electronic drift. Figure 2 shows the result, which is negligible. Custom current to voltage converters are being designed ${ }^{4}$, these will be integrated into the ALS control system. 


\section{CALIBRATION AND PERFORMANCE}

Each monitor is equipped with two motors for motion over several millimeters transverse to the beam, for positioning and calibration. During operation, a calibration sequence is performed by moving the monitor $100 \mu \mathrm{m}$ or so through the beam and observing the change of the signals to determine the sensitivity to beam motion. Empirical correction factors can be determined to minimize cross talk between the horizontal and vertical directions for the $45^{\circ}$ monitor ${ }^{3}$. If the signals from the upper blades are ' $a$ ' and ' $b$ ' and the lower blade signals are ' $c$ ' and ' $d$ ', the signal for vertical position at the detector is given simply by :

$$
\text { vertical position }=s^{*}\left(\alpha^{*} a+\beta^{*} b-\gamma^{*} c-\delta^{*} d\right) /\left(\alpha^{*} a+\beta^{*} b+\gamma^{*} c+\delta^{*} d\right)
$$

where $\alpha, \beta, \gamma$ and $\delta$ are numbers close to unity to minimize cross talk by compensating for unequal quantum efficiencies and amplifier gain, $s$ is the vertical sensitivity ( $s \approx 2000 \mu \mathrm{m}$ for the first monitor, $3500 \mu \mathrm{m}$ for the second). The zero of beam position is arbitrary in that it depends on the detector position. This normalization gives negligible error as the electron beam intensity decays.

The sensitivity depends on the undulator gap because varying the gap changes the geometrical pattern of illumination at the detector. The detectors become less sensitive to horizontal motion when the $K$ value is large at small gaps.

The detector may be deceived by the changing illumination as the undulator gap changes and may register the varying gap as apparent beam motion 5 . This effect is minimized by careful positioning of the detector in the beam. The residual gap-dependent position offset is then observed by recording data from the monitors as the undulator gap is changed. (There may be some real motion of the undulator beam hidden by this varying offset). The best operating beam position for the beamline can be determined from the optimum monochromator through-put at various undulator gaps An empirical gap-dependent offset correction can be found. The apparent beam motion arising from the gap-dependent offsets is shown in figure 3.

At present the monitors are most useful in providing a check for beam position drift. With a certain value of the undulator gap the normalized position signal gives an immediate measure of the beam position drift. In beamline 7.0, small drifts $(<200 \mu \mathrm{m})$ can be compensated by adjusting the first mirror. 


\section{STABILITY OF THE FACILITY}

Here we show some ALS stability measurements. Vibrational stability is excellent, within $10 \%$ of the r.m.s. electron beam size and divergence. Figure 4. shows the spectrum of the vertical motion measured at one of the monitors. Acoustic frequency oscillations are at frequencies from $10 \mathrm{~Hz}$ to $100 \mathrm{~Hz}$ and give an r.m.s. amplitude of vibrational motion at the monitor of about $4 \mu \mathrm{m}$.

Slow drift is negligible for the duration of a typical experiment. Figure 5. shows the vertical position and angle of the electron orbit at the center of the undulator for beamline 7.0, measured at $1 \mathrm{~Hz}$ over a period of several hours of normal operation. None of the undulators were changed during this time. The twelve minute oscillations are due to water temperature fluctuations with a measured amplitude of $\pm 0.5^{\circ} \mathrm{C}$.

The observed level of drift of the electron beam should have little effect on the beamline through-put. Figure 5. also shows the variation of the flux at the end of the beamline and confirms this. It is hard to see a clear correlation between through-put variation and beam motion at this level.

Figure 6. shows the orbit position measurement with the undulator in the adjacent straight section closing. Closed orbit compensation is available from an empirically determined look-up table to correct the gap dependent steering of this undulator. These measurements were made with and without compensation. The resulting beam motion is reduced from $200 \mu \mathrm{m}$ to $20 \mu \mathrm{m}$. (These measurements were made with prototype electronics, the fluctuations in the data over intervals of a few seconds are not real).

\section{FUTURE DEVELOPMENTS}

These monitors work, but they are tricky to use correctly. Presently no undulator beamline at the ALS requires better than the intrinsic orbit stability. This situation may change, at which point some collaboration will be required between the experimenters at the beamline and the ALS staff, to calibrate the monitors and determine precise offset so that they can be used in a feedback loop. In monochromator beamlines with an entrance slit the first mirror can be pitched slightly to correct for orbit shifts up to about $100 \mu \mathrm{m}$, beyond this the focussing of the first rinirror may be compromised and an orbit correction is then required. Future UV and soft $X$-ray undulator beamlines at the ALS will capitalize on the intrinsic orbit stability and will use entrance slit-less monochromators. For these designs the orbit will probably be actively stabilized against thermal drift. Active stabilization systems at acoustic frequencies seem not to be needed, vibrations seem negligible. 


\section{ACKNOWLEDGMENTS}

Thanks to Tom Swain for the thermal analysis of the monitors. The stable performance of the ALS is due to excellent work over several years by a large group of people in the accelerator group. Closed orbit compensation algorithms were implemented by Hiroshi Nishimura. Vibration data were analyzed by Greg Portmann.

This work was supported by the Director, Office of Energy Research, Office of Basic Energy Sciences, Materials Sciences Division of the U.S.Department of Energy, under Contract No. DE-AC03-76SF00098

\section{REFERENCES}

1. E. D. Johnson and T. Oversluizen, Rev. Sci. Instr., 60 (1989) 1947.

2. T. Warwick, P. Heimann, D. Mossessian, W. McKinney and H. Padmore, these proceedings

3. T. Warwick, D. Shu, B. Rodricks and E.D. Johnson, Rev. Sci. Instr., 63 (1992) 550.

4. J. Hinkson and M. Fahmie, private communication.

5. T. Mitsuhashi, A. Ueda and T. Katsura, Rev. Sci. Instr., 63 (1992) 534.

\section{FIGURE CAPTIONS}

Figure 1. Cutaway view of the first photon position monitor, showing the blades protruding through the bias plates towards the photon beam.

Figure 2. Simulated currents from a battery are used to generate a position signal to check amplifier drift, which is seen to be negligible.

Figure 3. Apparent source motion from the gap-dependent monitor offset, observed by recording daia from the monitors as the undulator is changed discretely from one constant gap to another.

Figure 4. Power spectrum of vibrational motion of the undulator beam at the second monitor, $12.6 \mathrm{~m}$ from the source. The r.m.s. amplitude is $4.1 \mu \mathrm{m}$.

Figure 5. Stability of the orbit and of the monochromator through-put at beamline 7.0 during steady operation of the ALS. Measurements were made at $1 \mathrm{~Hz}$. No undulators were changed and no orbit stabilization systems were active. 
Figure 6. Orbit stability measured at beamline 7.0 during operation with the adjacent undulator closing and opening, with and without dynamic closed orbit compensation for the gap-dependent steering of the undulator. 


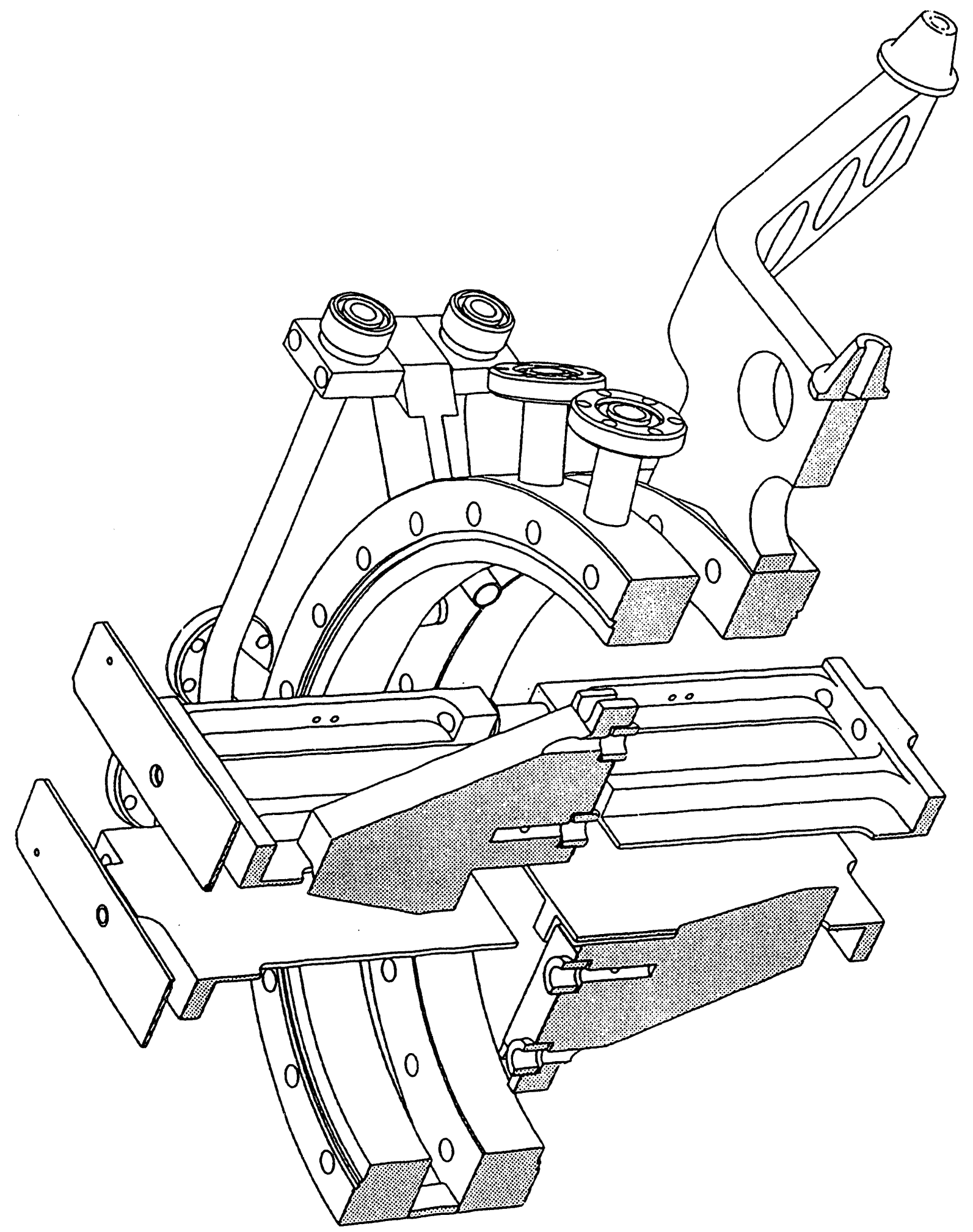

XBL 947-485 


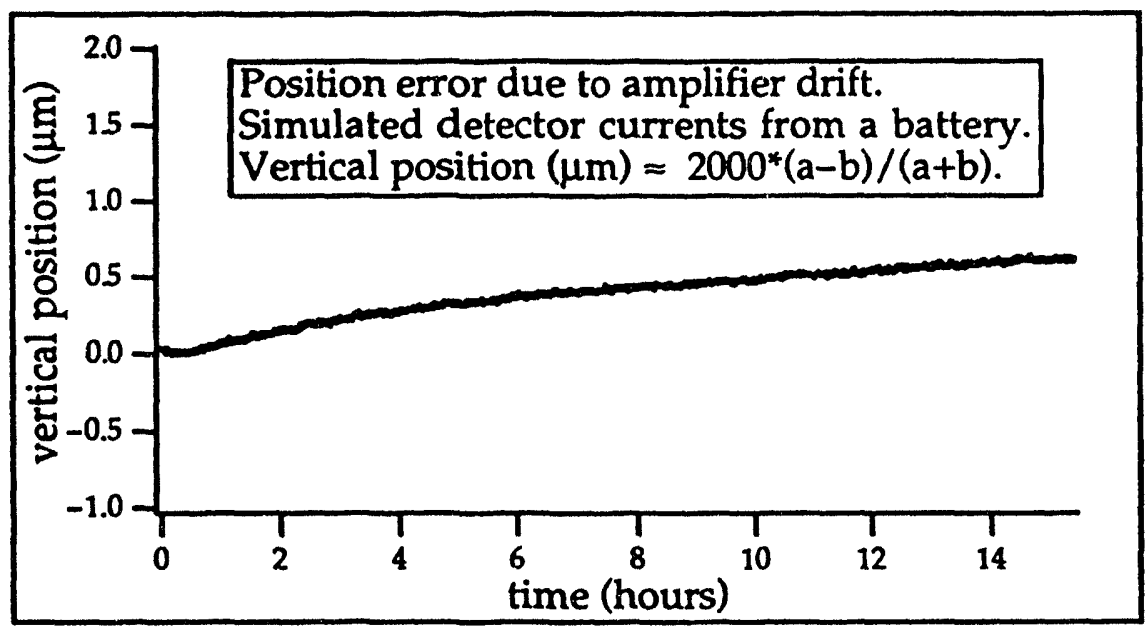



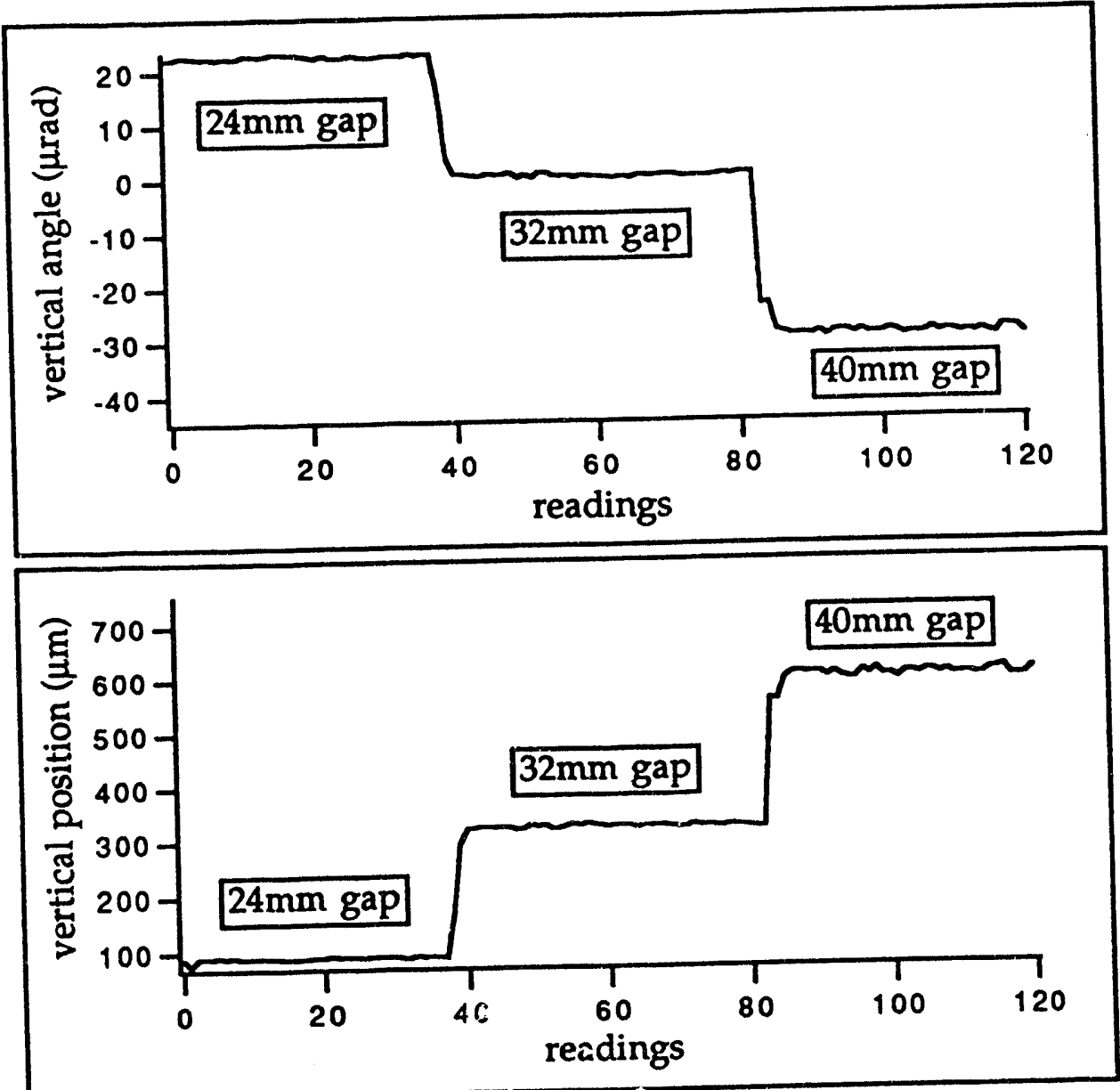


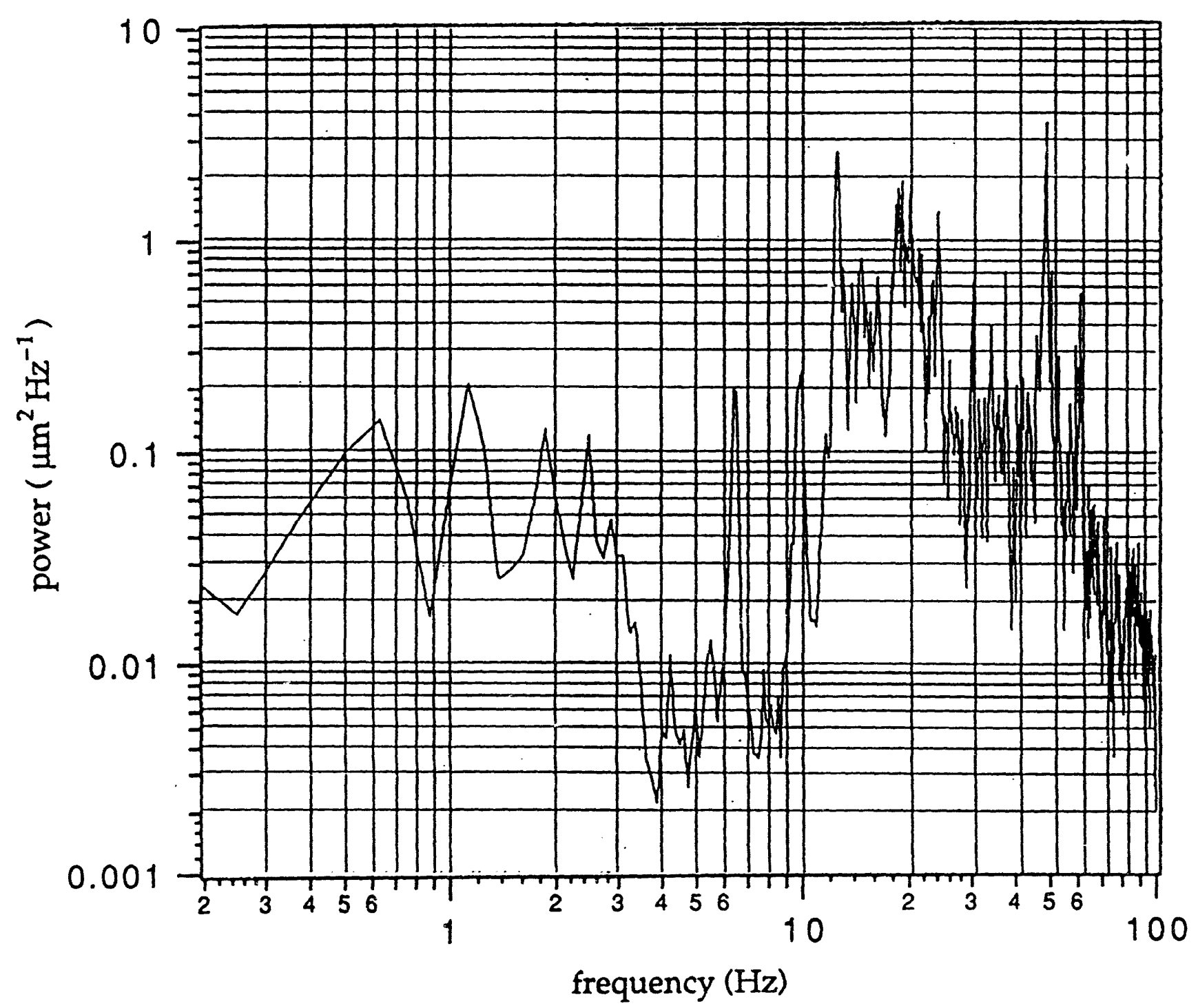



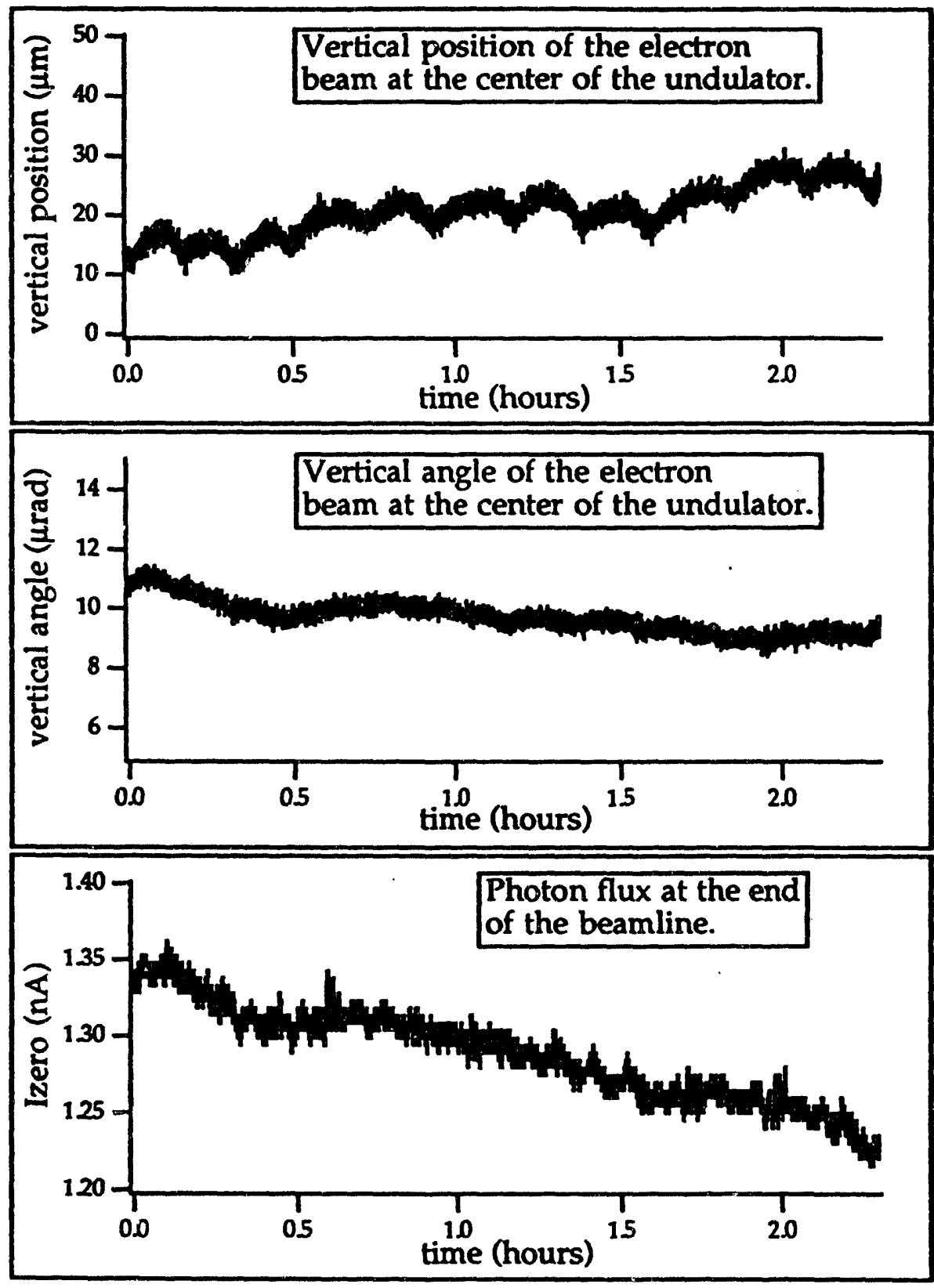


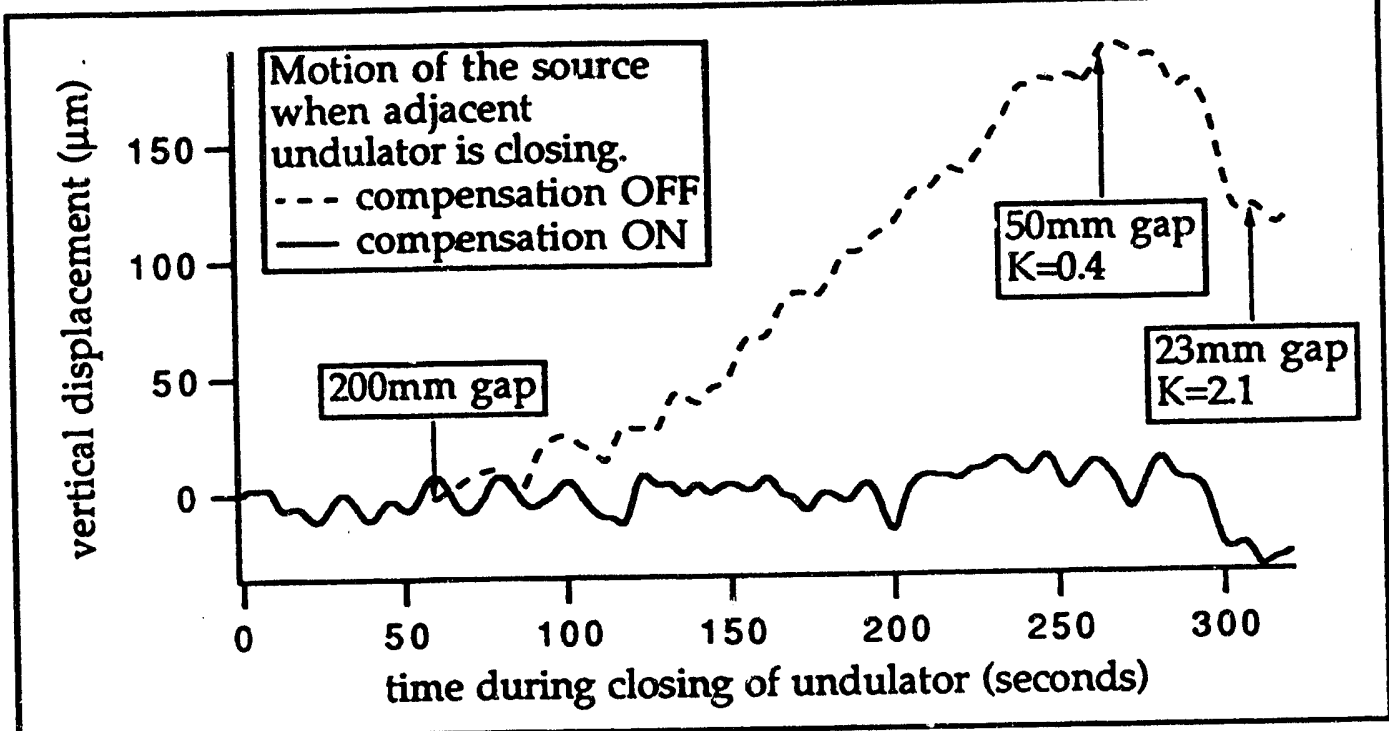



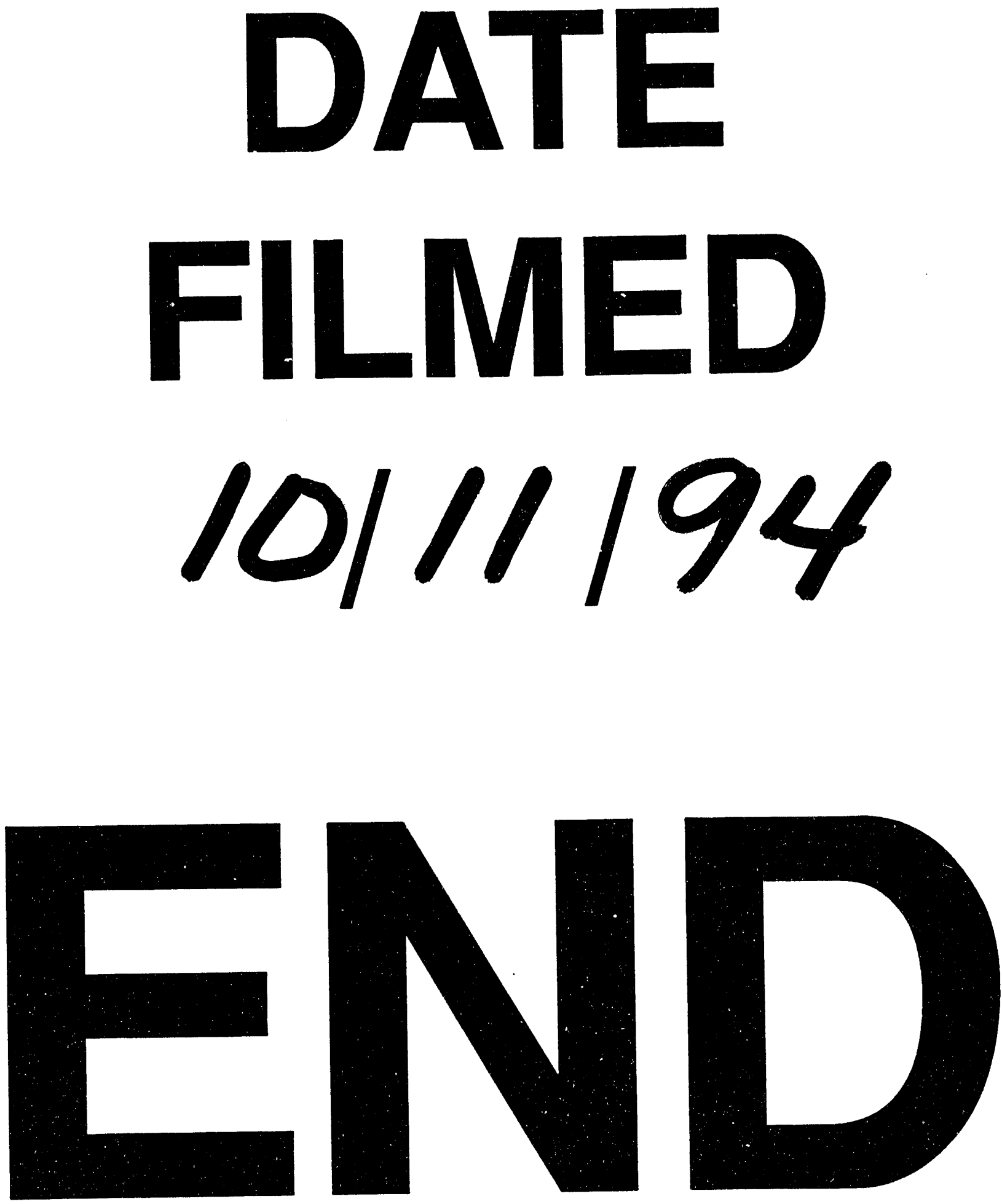
